\title{
MLS-assisted validation of WorldView-2 panchromatic image for estimating Pinus sylvestris crown height
}

\author{
Yi Lin ${ }^{1}$, Geoff West ${ }^{2}$, David Belton ${ }^{2}$, Petra Helmholz ${ }^{2}$ \\ ${ }^{1}$ Institute of Remote Sensing and Geographic Information System, Peking University, \\ 100871 Beijing, China \\ ${ }^{2}$ Cooperative Research Centre for Spatial Information (CRCSI), Department of Spatial \\ Sciences, Curtin University of Technology, 6708 Perth, Australia \\ * the corresponding author: yi.lin@pku.edu.cn
}

Short biographical notes:

Yi Lin, Dr., Research Professor, received his PhD in Photogrammetry and Remote Sensing from Peking University in 2009. He has authored/co-authored more than 20 peer-reviewed journal papers.

Geoff West, Dr., Professor of Spatial Information, received his PhD in Systems Engineering from City University, London in 1982. He has authored/co-authored 55 peer-reviewed journal papers and 191 peer-reviewed conference papers.

David Belton, Dr., Research Fellow, received his PhD in Surveying and Photogrammetry (Laser Scanning) from Curtin University in 2008. He has authored/co-authored more than 20 peerreviewed conference and journal papers.

Petra Helmholz, Dr., Lecturer, received her Dr.-Ing. (PhD) in Photogrammetry from the Leibniz University Hannover, Germany in 2012. She is an early carrier researcher and has published over 18 papers. 


\title{
MLS-assisted validation of WorldView-2 panchromatic image for estimating Pinus sylvestris crown height
}

\begin{abstract}
High spatial resolution satellite imaging has the advantages of both fine scale and large coverage that indicates the potential for measuring forest morphologies. However, because of the aerial view, imaging has limited capacity of explicitly deriving the under-crown structural parameters. A possible solution is to explore the relationships between variables such as crown height and the feature parameters readily derived from the satellite images. However, field sampling of the training data is not a trivial task. To handle this issue, this study explored the state-of-the-art remote sensing technology of vehicle based mobile laser scanning (MLS) for collecting the sample data. Evaluation for the case of the Scots Pine (Pinus sylvestris) trees has preliminarily validated the plan. That is, information from MLS enabled, the parameter of crown height to be correctly estimated from WorldView-2 panchromatic images.
\end{abstract}

Keywords: Crown height estimation; WorldView-2 panchromatic image; Mobile laser scanning (MLS)

Subject classification codes:

\section{Introduction}

With the advantages of both fine scale and large cover, high spatial resolution satellite imaging is a promising choice for measuring morphology ranging from single trees up to a full forest. A number of investigations have been carried out on this efficient data collection technique to obtain different structural parameters of trees for large areas (Grote, 2003; Beguet et al., 2012). For instance, multi-scale segmentation of QuickBird images was carried out for exploring forest structural complexity (Lamonaca et al., 2008), and the texture parameters derived from WorldView-2 (WV-2) multispectral images were applied for predicting forest structural parameters such as basal area and stem volume (Ozdemir and Karnieli, 2011). Specifically for tree-level structure determination, satellite image bands with higher spatial resolutions such as WV-2 
panchromatic images (Ozdemir and Karnieli, 2011) show promise because of their ability to supply more structural details.

However, , determining tree structural attributes under the crown e.g. trunk and branch diameter, is difficult for high spatial resolution satellite imaging because the tree canopy obstructs the view. Such a case is Crown Height $(\mathrm{CH})$, which is defined as the height from the ground to the point of attachment of the lowest living branch to the trunk. $\mathrm{CH}$ as it can indicate tree growth and tree timber quality (Petersson, 1997). This is of ecological and economic significance and hence precise acquisition of $\mathrm{CH}$ is needed. Determining the relationships between $\mathrm{CH}$ and the feature parameters readily derived from high spatial resolution satellite images is a potential solution (Barbier et al., 2012).

However, field sampling of the training data proves to be a non-trivial task (Lamonaca et al., 2008), and it is hypothesized that the state-of-the-art 3D remote sensing technology: Mobile Laser Scanning (MLS) is a potential solution. In contrast to manual measurement, photography (Macfarlane et al., 2007) and static Terrestrial Laser Scanning (TLS) (Yang et al., 2013), MLS can measure many individual trees in a more efficient and labour-saving way. This is because the scanning occurs while the vehicle is moving at a reasonable speed and covering large areas. This strength means that more samples can be measured within the same time span, and more samples means that higher accuracy and robustness can be obtained for parameter estimation. This aligns with the suggestions by botanists and ecologists of using diverse data sources for tree characterization (Heinzel and Koch, 2012). MLS has been applied to tree modeling (Rutzinger et al., 2010) and crown reconstruction (Lin and Hyyppä, 2012). However, there have been no attempts on using MLS to supply the sample data of individual trees 
as ground truth for calibrating the parameter estimations from WV-2 panchromatic images.

To fill this technical gap, this study explored how to introduce MLS into the task of validating high spatial resolution satellite images for tree $\mathrm{CH}$ estimation. Here, the tree species of concern is Scots Pine (Pinus sylvestris), and the specific satellite images are the typical WV-2 panchromatic images ( $0.46 \mathrm{~m}$ ground sampling distance (GSD) and $16.4 \mathrm{~km}$ swath width at nadir).

\section{Data}

The test site is located in the north-west corner of Curtin University, Perth, Australia

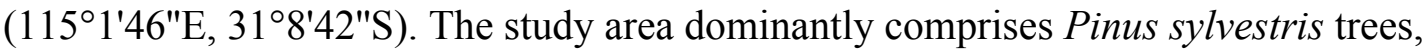
which grow naturally without their crowns being trimmed or pruned. The overlapping WV-2 panchromatic image collected on 3 December 2011 and published with its Ground Sample Distance (GSD) uniformly set to $0.5 \mathrm{~m}$ is shown in Figure 1(a).

The 3D laser sampling was done with a MDL Dynascan S250 MLS system mounted on a car on the $1^{\text {st }}$ May 2013. The MLS has a nominal range of $250 \mathrm{~m}$, and can capture 36,000 points $/ \mathrm{sec}$ at a scan-head rotation rate of $20 \mathrm{~Hz}$. The relative point accuracy of the laser is in the range of $+/-1 \mathrm{~cm}$. An aerial view of the acquired point clouds representing the test site is shown in Figure 1(b) (The darker the pixels, the higher above the ground). This is registered with the WV-2 panchromatic image, which has undergone geo-referencing before its commercial distribution.

Note that the temporal interval of one and a half years between the two data collections only has a small influence on the parameter estimations, because the Pinus sylvestris trees are evergreen and there are only small changes in terms of crown height and crown cover. The data registration was implemented by manually extracting the control points from the two datasets. The individual trees were extracted from the WV-2 
panchromatic imagery by manually drawing regions-of-interest (ROIs) using the ENVI software environment ${ }^{1}$. For the MLS data manually isolating groups of 3D points was carried out using the Leica Cyclone software environment ${ }^{2}$. Table 1 shows statistical information for the 37 Pinus sylvestris trees extracted from the MLS-collected 3D point clouds that have reasonably complete representations. The statistics are possible because each 3D point has 3D coordinates and hence, for example, it is easy to determine the maximum height of a tree from the 3D point with the highest height value.

\section{Methods}

The analysis consisted of three steps: first, the $\mathrm{CHs}$ as references were derived from the MLS data; second, feature parameters were extracted from the WV-2 panchromatic image; and finally, the relationships between the MLS-derived CHs and the imagederived feature parameters were explored and the image-based $\mathrm{CH}$ prediction formulas established.

Figure $2 \mathrm{a}$ shows the tree form and its presentation in the WV-2 panchromatic image are indicated in Figure 2(a) illustrates an example of a Pinus sylvestris tree along with definitions of the required parameters. Figure 2(b) diagrammatically shows a side view approximation to a crown, modelled as a paraboloid with a circular base, with the dots showing the pixels at their correct heights above the ground. The lack of correspondence of height to pixel grey level is illustrated i.e. there is an inconsistency in reflectance spatial distribution for individual crowns. Figure 2(c) displays the irregularity of the vertical projection shapes on the ground compared with a circular model. The irregularity is mainly because of variations in tree shape but also slightly

\footnotetext{
${ }^{1}$ http://www.exelisvis.com/ProductsServices/ENVIProducts/ENVI.aspx

${ }^{2}$ http://hds.leica-geosystems.com/en/Leica-Cyclone_6515.htm
} 
because of oblique or off vertical viewing angle. Determining the correspondence between the various parameters is addressed in the following sections.

\subsection{Determining tree crown height from MLS data}

The MLS data was used to estimate the crown height as shown in Figure 1 from by finding the lowest branches guided by analysis of the tree trunk for the many trees in the data. It is common to quantify a tree by the diameter of the trunk $1.37 \mathrm{~m}$ above the ground so determining this position was the first step. The location of the ground is easily extracted from the MLS data as the lowest points in the data when there are many points at the same location as expected for trees. The horizontal layer of 3D points covering the common variable of Diameter at Breast Height (DBH) i.e. $1.37 \mathrm{~m}$ in the MLS data is segmented out and projected onto a horizontal plane. Next the points are clustered and the centres of each cluster deemed as the possible locations of the trunks. Cluster analysis works because the distances between the points for each tree are much smaller than those between the trees. For each cluster, a vertically aligned cylinder is generated. The height of the cylinder is determined from the highest $3 \mathrm{D}$ point above each cluster hypothesized to be the top of the tree and set to $50 \%$ of the height. 3D points near to the cylinder are hypothesized to be on the trunk and used to determine the correct trunk axis using principal component analysis (PCA) (Monnier et al., 2012). Finally, the cylinder is aligned with the computed trunk axis and used to search upwards for the location of the lowest living branch identified by comparing the distance of each 3D point to the trunk axis to a preset threshold. The first $3 \mathrm{D}$ point further than the threshold is taken to indicate the start of the lowest branch.

PCA find the trunk axis as follows. PCA uses an orthogonal transformation to convert a set of observations of possibly correlated variables into a set of values of linearly uncorrelated variables called principal components (Monnier et al., 2012). The 
resulting first principal component has the largest possible variance, and each succeeding component is orthogonal to the preceding components and has decreasing variance (Monnier et al., 2012). For the MLS 3D points of trees, the most variance is along the approximately vertical axis of the trunk and the described by the first principal component (Bucksch et al., 2009). The two principal components with lower variances describe the variation across the trunk perpendicular to the trunk axis.

\subsection{Tree top location in WorldView-2 panchromatic images using ellipsoid}

\section{fitting}

As illustrated in Figure 2(b), the high spatial resolution makes trees in the WV-2

panchromatic image appear different to that in conventional low spatial resolution satellite images. In low resolution satellite images, each crown is typically characterized by one bright pixel or a single grouping or cluster of several bright pixels (e.g., Wang et al., 2004). In the WV-2 panchromatic image, there can be many isolated bright pixels or a number of separate clusters producing many possible crown tops for each tree.

Thereby, the traditional local maximum seeking methods (Ke and Quackenbush, 2011) introduces spurious crown tops.

However, the spatial distribution of the reflectance for each individual tree still follows the mountain-shaped model (Ke and Quackenbush, 2011). Hence, an ellipsoidfitting-based tree top location method is proposed here as indicated by the model in Figure 2(b). The ellipsoid-characterizing formula is

$$
\frac{\left(x-x_{0}\right)^{2}}{a^{2}}+\frac{\left(y-y_{0}\right)^{2}}{b^{2}}+\frac{\left(z-z_{0}\right)^{2}}{c^{2}}=1
$$

where $x$ and $y$ correspond to the location of each pixel in the image, and $z$ is related to the brightness value. The coefficients are determined by least squares fitting (Bertoni, 
2010). The computed variables $x_{0}$ and $y_{0}$ of the best fit ellipsoid to the brightness values of each crown are the centre of the ellipsoid indicating the location of the tree top.

\subsection{Feature parameters extraction and regression analysis}

As shown in Figure 2(c), it is hard to directly use the crown shapes from the images to predict the parameter $\mathrm{CH}$. Instead, some feature parameters capable of describing the structural specialties of crowns are extracted as the independent variables for prediction. The longest spread (LS) and the longest cross-spread (LCS) as marked in Figure 2(c) are used, since they can characterize the long, stout branches which make tree crowns asymmetrical, and the dominant branches respectively. Note that although crown spread is usually taken independent of trunk position (Blozan, 2006), LS and LCS in this study are defined to pass through a tree top. In this way, the outlier errors introduced by some branches that are sticking out can be overcome to some extent. In addition, the circle with an area the same as the cover of each crown is also determined, and its Equivalent Diameter (ED) is calculated as one feature parameter. The ED, in a statistical sense, is proposed to handle the issues caused by crown projection shape irregularity.

Correlation analysis is performed between each of the image-derived feature parameter sets and the MLS-derived $\mathrm{CH}$ set. Linear regression analysis after transformation of the response variable is used to determine their relationships, and the degree of correlation assessed using the coefficient of determination $\mathrm{R}^{2}$ (Zar, 2010) and the Root Mean Square Error (RMSE) (in its standard deviation form). The fitted equations by least squares fitting can then be used as the prediction formulas for estimating the $\mathrm{CH}$ parameter. 


\section{Results}

\subsection{Crown heights derived from the MLS data}

The results of $\mathrm{CH}$ extraction in the MLS point clouds respectively using the automatic cylinder-based algorithm and manually are shown in Figure 3. The mutual CHs present certain correlations, as indicated by the output $\mathrm{R}^{2}$ of 0.59 . The results show that MLS can serve as an efficient field sampling technique for supplying reasonably accurate tree attribute training data.

\subsection{Tree tops identified in the WorldView-2 panchromatic image}

The location of the tree top for each individual tree was determined in the WV-2 panchromatic image using the ellipsoid-fitting algorithm. The reference locations are the centres of the corresponding trunks extracted using MLS, as the growth of Pinus sylvestris trees tend to be monopodial. Figure 4 shows that the determined tree tops are generally biased towards the north-east orientation. The average deviation is $1.96 \mathrm{~m}$, but all of the extracted tree tops lie within the boundaries of the corresponding trees.

\subsection{Regression models between longest spread, longest cross-spread, equivalent diameter and crown height}

With the tree tops determined above, the parameters of LS and LCS were calculated using the PCA-based method. The ED for each crown was also calculated. Their correlations with the MLS-derived CHs are shown in Figure 5(a), Figure 5(b) and Figure 5(c) respectively. ED gives the best results, while LS gives the worst. LCS is better than LS and shows that the LS related long, stout branches which make tree crowns appear asymmetrical are unreliable for establishing the metrics for inferring other tree attributes. All of the results are low but all positive. The formulas for 
predicting CHs based on the WV-2 panchromatic image are also listed in Figure 5.

\section{Discussion and summary}

The results of automatic $\mathrm{CH}$ extraction from MLS data as shown in Figure 3 are positive but need to be improved. The reason is that the sampling density of the MLS system used was set too low, even though the vehicle was moving at a slow pace of approximately 20 kilometres/hour. The distance of $0.5 \mathrm{~m}$ between any two adjacent scan profiles is not enough to represent the structures of branches in detail. This is also one of the possible reasons for the $2.42 \mathrm{~m}$ offset existing in the formula as listed in Figure 3. Another possible reason is that the tiny lowest living branches are easily missed in the process of $\mathrm{CH}$ automatic extraction, which is not true for manual extraction. The results can be improved by increasing the sampling density by making the vehicle travel more slowly (if practical so as not to disrupt other traffic), by combining multiple scans or vehicle passes, by using multiple scanners, or by carrying out the scanning in the stopand-go mode (Lin et al., 2013).

For the detected tree tops, there is an obvious bias towards the north-east direction as shown in Figure 4. The reasons for this are that sun passes from east to west to the north in the southern hemisphere and the data collection occurred before noon. In addition the prevailing west wind from the Indian Ocean tends to make the crowns in Perth easily deviate to the east. Under these conditions, the elevation angle of the sun tends to make parts of the tree canopy facing the sun have higher reflectance than the real tree top moving the the whole pattern of crown reflectance away from the tree top. The temporal model of sunlight incidence variation can be incorporated to rectify this, given that the satellite and MLS systems accurately record the time and location of the data acquisition for each tree. 
The comparisons Figure 5 indicate that the feature parameter ED in a statistical sense is the best choice. This fits experience obtained from the satellite-image-based forest inventory studies. That is, although the WV-2 panchromatic images can show $0.5 \mathrm{~m}$ GSDs, it is still hard to directly extract the structural parameters of individual trees at high accuracies. Instead, satellite-based tree mapping in practice is generally aimed at determining the overall tree attributes from the perspective of many trees or large coverage. The variable ED acquired from WV-2 panchromatic images is more appropriate for $\mathrm{CH}$ derivation, and in particular for large areas, samples of high volumes can eliminate the effect of relatively-high errors for individual trees. This is promising for stem volume estimation (Ozdemir and Karnieli, 2011) in forest management.

As regards the positive but far-from-perfect performances as shown in Figure 5, the above-mentioned discussions for improvements can help. Investigations such as proposed by Peper et al., 2001 need to be conducted, such as exploring more complicated equations capable of delineating the relationships between $\mathrm{CH}$ and $\mathrm{ED}$. Tests showed that when transforming the response variable (e.g. by taking logs), $\mathrm{R}^{2}$ can be improved (for Figure 5(c) from 0.052 to 0.066). In addition, more refined models will benefit from more data acquisition i.e. more trees to be scanned using more refined MLS scanning methods. Many other factors can be taken into account for $\mathrm{CH}$ prediction improvement. Feature parameters such as texture (Ozdemir and Karnieli, 2011) can be extracted and their individual or composite correlations with $\mathrm{CH}$ analyzed and compared to determine the more accurate $\mathrm{CH}$ prediction formulas. Tree competition (Contreras et al., 2011) is another element of interest. In fact, for several trees tightly clustered together, the tree with the smallest crown width has similar $\mathrm{CH}$ as the other trees. If this tree is lower than the other trees, it cannot receive enough sunlight. Its small size is mainly due to limited crown-expanding space rather than small crown 
height. Thereby, some competition-associated compensation coefficients need to be added.

Overall, this preliminarily has shown the usefulness of the combination of MLS and WV-2 panchromatic imaging for tree-level $\mathrm{CH}$ estimation in the case of Pinus sylvestris trees. MLS proved to be applicable for acquiring the training data for $\mathrm{CH}$, and WV-2 panchromatic images suggested the possibility of effective prediction of tree $\mathrm{CH}$, or tree structural parameters in a broader sense, for large areas.

\section{Acknowledgements}

This work is supported by the Australian Endeavour Research Fellowships programme.

\section{References:}

Barbier, N., Couteron, P., Gastelly-Etchegorry, J.P., Proisy, C., 2012, Linking canopy images to forest structural parameters: potential of a modeling framework. Annals of Forest Science, 69, pp. 305-311.

Beguet, B., Chehata, N., Boukir, S., Guyon, D., 2012, Retrieving forest structure variables from very high resolution satellite images using an automatic method. ISPRS Annals of the Photogrammetry, Remote Sensing and Spatial Information Sciences, I-7, pp. 1-6.

Bertoni, B., 2010, Multi-dimensional ellipsoidal fitting. Department of Physics, South Methodist University, Technical Report No. SMU-HEP-10-14.

Blozan, W., 2006, Tree measuring guidelines of the Eastern Native Tree Society. Bulletin of the Eastern Native Tree Society, 1, pp. 3-10.

Bucksch, A., Lindenbergh, R., Menenti, M., 2009, Applications for point cloud skeletonizations in forestry and agriculture. Proc. SPIE. 2009, Article no. 7460.

Contreras, M.A., Affleck, D., Chung, W., 2011, Evaluating tree competition indices as predictors of basal area increment in western Montana forests. Forest Ecology and Management, 262, pp. 1939-1949.

Grote, R., 2003, Estimation of crown radii and crown projection area from stem size and tree position. Annals of Forest Science, 60, pp. 393-402.

Heinzel, J., Koch, B., 2012, Investigating multiple data sources for tree species classification in temperature forest and use for single tree delineation. 
International Journal of Applied Earth Observation and Geoinformation, 18, pp. $101-110$.

Ke, Y., Quackenbush, L.J., 2011, A review of methods for automatic individual treecrown detection and delineation from passive remote sensing. International Journal of Remote Sensing, 32, pp. 4725-4747.

Lamonaca, A., Corona, P., Barbati, A., 2008, Exploring forest structural complexity by multi-scale segmentation of VHR imagery. Remote Sensing of Environment, 112, pp. 2839-2849.

Lin, Y., Hyyppä, J., 2012, Multiecho-recording mobile laser scanning for enhancing individual crown reconstruction. IEEE Transactions on Geoscience and Remote Sensing, 50, pp. 4323-4332.

Lin, Y., Hyyppä, J., Kukko, A., 2013, Stop-and-go mode: Sensor manipulation as essential as sensor development in terrestrial laser scanning. Sensors, 13, 81408154.

Macfarlane, C., Grigg, A., Evangelista, C., 2007, Estimating forest leaf area using cover and fullframe fisheye photography: Thinking inside the circle. Agricultural and Forest Meteorology, 146, pp. 1-12.

Monnier, F., Vallet, B., Soheilian, B., 2012, Trees detection from laser point clouds acquired in dense urban areas by a mobile mapping system. ISPRS Annals of the Photogrammetry, Remote Sensing and Spatial, Information Sciences, I-3, pp. $245-250$.

Ozdemir, I., Karnieli, A., 2011, Predicting forest structural parameters using the image texture derived from WorldView-2 multispectral imagery in a dryland forest, Israel. International Journal of Applied Earth Observation and Geoinformation, 13, pp. 701-710.

Peper, P.J., McPherson, E.G., Mori, S.M., 2001, Equations for predicting diameter, height, crown width, and leaf area of San Joaquin valley street trees. Journal of Arboriculture, 27, pp. 306-317.

Petersson, H., 1997, Functions for predicting crown height of Pinus sylvestris and Picea abies in Sweden. Scandinavian Journal of Forest Research, 12, pp. 179-188.

Rutzinger, M., Pratihast, A.K., Elberink, S.O., Vosselman, G., 2010, Detection and modelling of 3D trees from mobile laser scanning data. International Archives of Photogrammetry, Remote Sensing and Spatial Information Science, 38, pp. 520525. 
Wang, L., Gong, P., Biging, G.S., 2004, Individual tree-crown delineation and treetop detection in high-spatial-resolution aerial imagery. Photogrammetric Engineering \& Remote Sensing, 70, pp. 351-357.

Yang, X., Strahler, A., Schaff, C., Jupp, D., Yao, T., Zhao, F., Wang, Z., Culvernor, D.,Newnham, G., Lovell, J., Dubayah, R., Woodcock, C., and Ni-Meister, W., 2013, Three-dimensional forest reconstruction and structural parameter retrievals using a ground-based full-waveform lidar instrument (Echidna®). Remote Sensing of Environment, 135, pp. 36-51.

Zar, J.H., 2010, Biostatistical analysis (5th edition). Pearson Prentice-Hall: Upper Saddle River, 944 pp. 
Table 1. The statistical characteristics of the Pinus sylvestris trees from the MLS data.

\begin{tabular}{c|cccc}
\hline & Max & Min & Mean & Std \\
\hline Tree height $(\mathrm{m})$ & 28.38 & 17.60 & 23.68 & 1.98 \\
Crown basal area $\left(\mathrm{m}^{2}\right)$ & 23.73 & 16.66 & 21.06 & 1.76 \\
Crown height $(\mathrm{m})$ & 18.95 & 9.29 & 14.60 & 2.05 \\
\hline
\end{tabular}

Figure 1. The WorldView-2 panchromatic image (a) and the MLS-collected point cloud (b) of the test site.

(a)

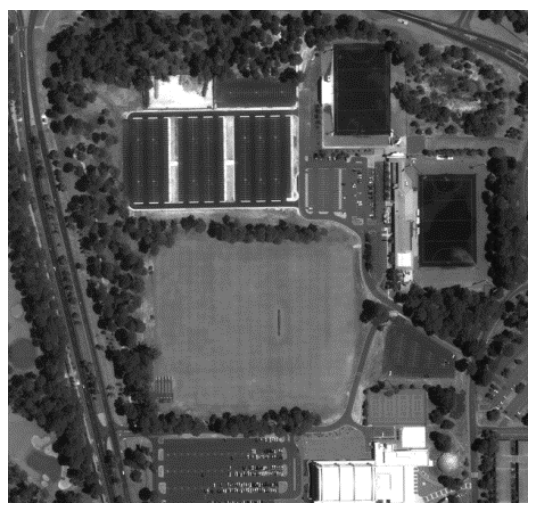

(b)

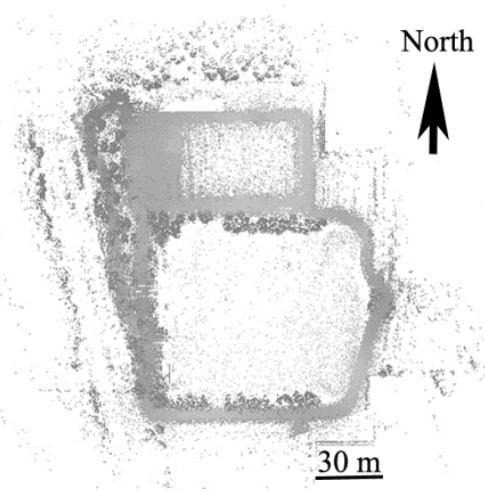

Figure 2. Illustrations of (a) Pinus sylvestris tree structure with the structural parameters,

(b) crown reflectance indicated by pixel brightness, and (c) the coverage and outline of a crown in the WorldView-2 image (The acronyms listed in the image are referred to in the text).

(a)

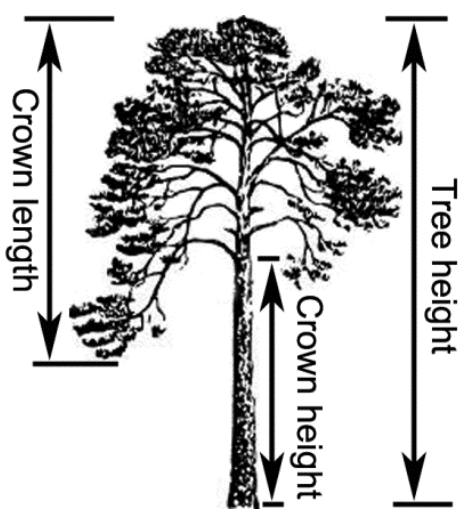

(b)

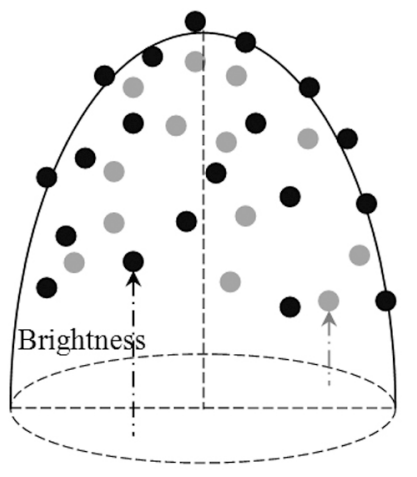

(c)

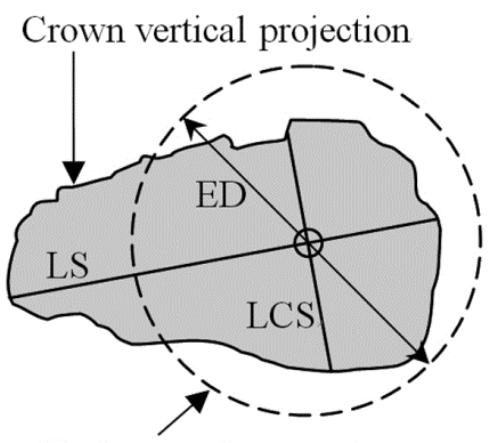

Circle covering equal area 
Figure 3. The regression model between the reference crown heights acquired by both manual and automatic extraction from the MLS data.

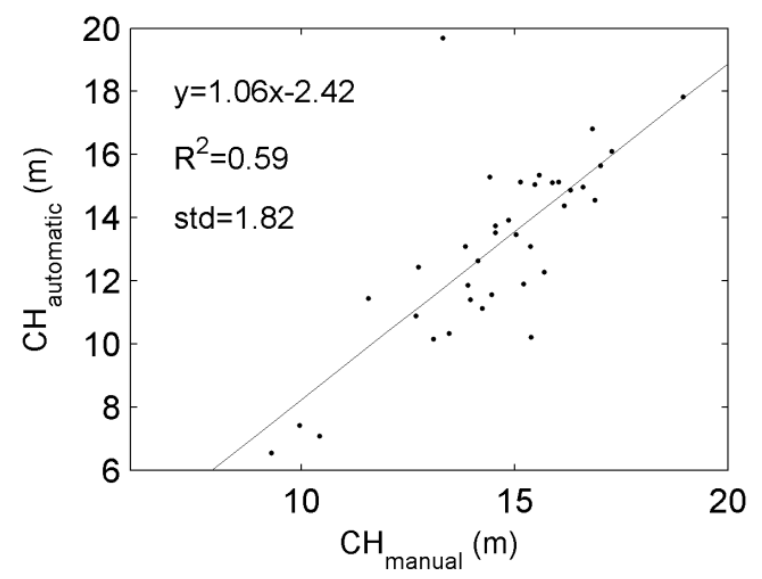

Figure 4. The deviations of the tree top locations inferred from the WorldView-2 image compared to the reference ones (reference locations transformed to $(0,0)$ ).

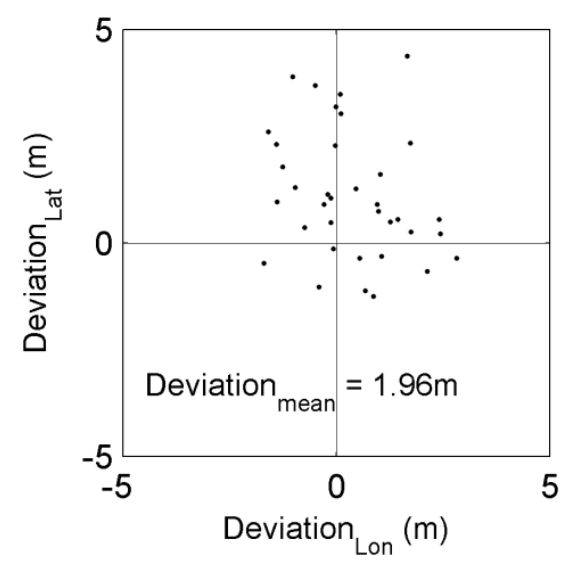


Figure 5. The regression models between the crown heights $(\mathrm{CH})$ and (a) the longest spreads (LS), (b) the longest cross-spreads (LCS), and (c) the equivalent distances (ED).
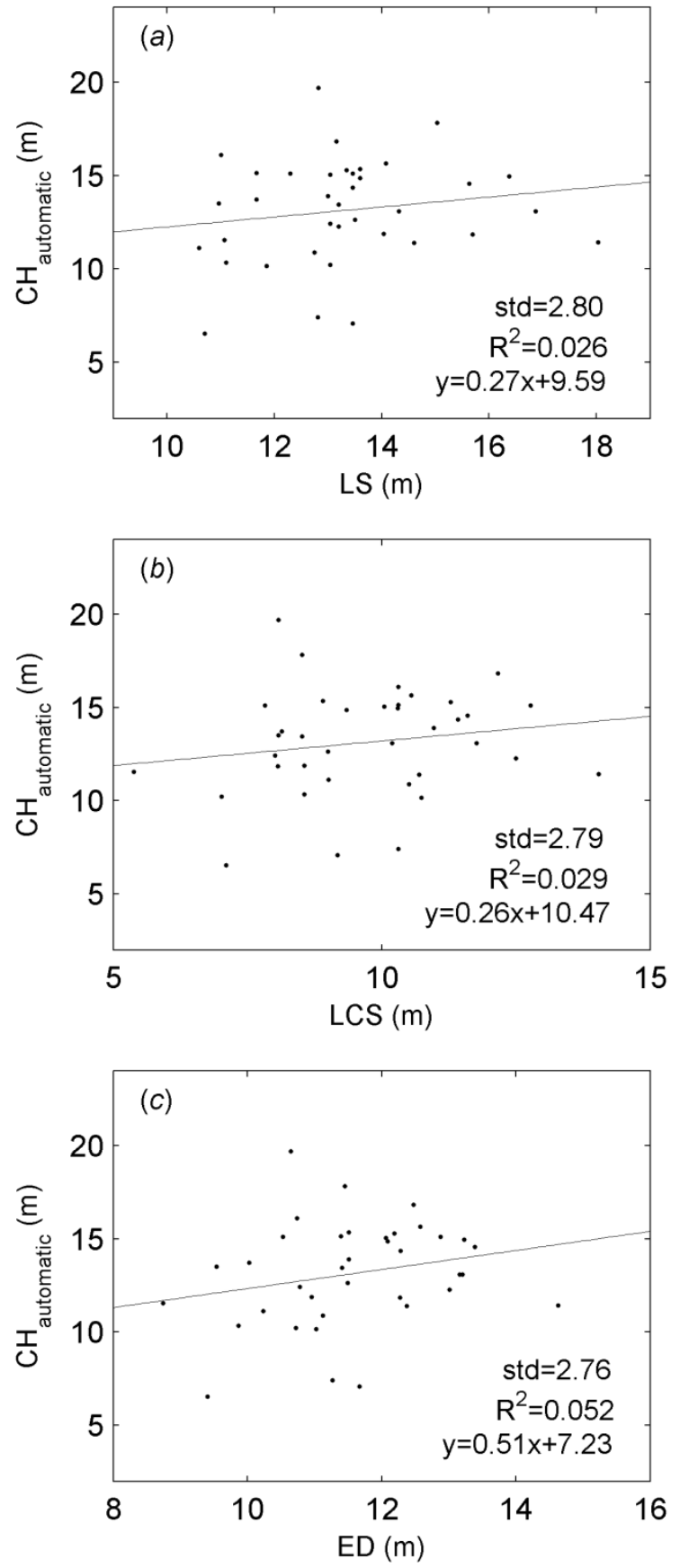